\title{
Analysis and Implementation of an Ultra-Wide Tuning Range CMOS Ring-VCO With Inductor Peaking
}

\author{
Ke Li, Fanfan Meng, Dave J. Thomson, Peter Wilson, and Graham. T. Reed
}

\begin{abstract}
A novel ring voltage controlled oscillator (VCO) topology is proposed which uses monolithic inductors as a peaking load. Four design examples have been fabricated and tested to verify the proposed circuit structure. The highest measured oscillation frequency is $25.07 \mathrm{GHz}$, with a tuning range of more than four octaves, and the active area is $0.0085 \mathrm{~mm}^{2}$. The design has the highest combined frequency and tuning range with the best figure of merit $(\approx 195)$ comparable to previously published work.
\end{abstract}

Index Terms-Delay cell, inductor peaking, ring oscillator, VCO.

\section{INTRODUCTION}

A $S$ DATA traffic continually increases, the date rate in wireline transceivers has exceeded $25 \mathrm{~Gb} / \mathrm{s}$ [1]. Traditionally, all the clock generation systems [Phase-Locked Loop (PLL) and Clock Data Recovery (CDR)] in these transceivers are based on LC (Inductor-Capacitor) tank VCOs. The main reason for this is that LC-VCOs have inherently excellent phase noise performance and usually a relatively high oscillation frequency, which make them appropriate for wireless and optical transceivers [2], [4]. However, one significant drawback with LC-VCOs is that they have a relatively small tuning range, potentially reducing the data-rate range that a transceiver can operate at. In contrast to the LC based Oscillators, Ring Oscillator (RO) based VCOs are attractive for digital applications and copper based wireline transceivers owing to their wider tuning range, ease of integration and small die area [2].

Although LC-VCOs and RO-VCOs are considered as opposite solutions due to the seemingly opposing features described previously, it is possible to take an innovative approach to combine the advantages of these two methods. This approach is inspired by previous work [3], which adopting several monolithic inductors with three stages of ring oscillator to achieve a terahertz frequency. In this work we therefore focus on an implementation of incorporating peaking inductors with a typical RO-VCO structure, to obtain a combination of ultrawide tuning range and small die area. To demonstrate the

Manuscript received July 13, 2016; revised September 19, 2016; accepted October 13, 2016. This work was supported by the UK Engineering and Physical Sciences Research Council (EPSRC) under the grant of "Silicon Photonics for Future Systems."

K. Li, F. Meng, D. J. Thomson, and G. T. Reed are with the Optoelectronics Research Centre, University of Southampton, Southampton SO17 1BJ, U.K.

P. Wilson is with the Department of Electronic and Electrical Engineering, University of Bath, Bath BA2 7AY, U.K.

Color versions of one or more of the figures in this paper are available online at http://ieeexplore.ieee.org.

Digital Object Identifier 10.1109/LMWC.2016.2629968

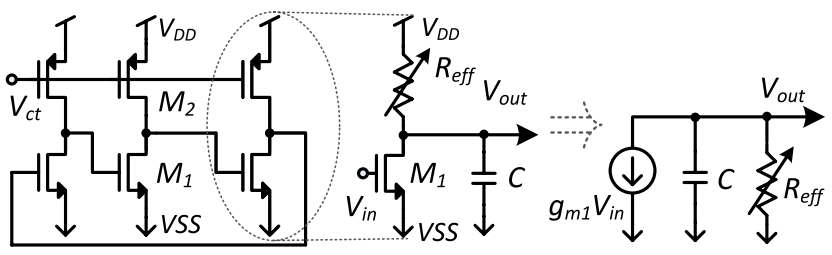

Fig. 1. Common source amplifier based RO-VCO.

functionality and advantages of the proposed circuit topology, four RO-VCO designs have been implemented using the $130 \mathrm{~nm}$ and $65 \mathrm{~nm}$ processes. As shown later in this letter, the VCO implemented on the $65 \mathrm{~nm}$ process can operate up to $25.07 \mathrm{GHz}$ with a tuning range of more than four octaves. The theoretical analysis of proposed RLC-RO-VCO is presented in section II. Four design examples are then presented in section III, associated with measurement results of each example. Finally, section IV concludes the letter.

\section{THEORETICAL ANALYSIS}

It is well-known that any RO must satisfy two of Barkhausen's criteria for oscillation, namely, the phase shift around the total feedback loop must be a multiple of $360^{\circ}$ at its oscillation frequency and the magnitude of the loop gain at that frequency must be unity [4].

Therefore for a typical three stage $\mathrm{RO}-\mathrm{VCO}$ as shown in Fig. 1, each delay cell can be treated as a common source amplifier, with its transfer function given as:

$$
H(s)=V_{\text {out }} / V_{\text {in }}=-g_{m 1} R_{\text {eff }} /\left(1+s C R_{\text {eff }}\right)
$$

The capacitance $C$ denotes all the capacitance associated at the $V_{\text {out }}$ node, and a variable resistor $R_{\text {eff }}$ is used to represent the effective resistance of the transistor M2, whose gate voltage is controlled by the external signal $V_{c t}$. From (1), it can be seen that $3-\mathrm{dB}$ bandwidth of this amplifier is $1 /\left(2 \pi R_{\text {eff }} C\right)$, and frequency tuning is realized by changing the effective resistance $R_{e f f}$ of transistor M2. In addition to the variable resistance $R_{\text {eff }}$, the gate capacitance $C$ in the load stage and the parasitic capacitance associated with wiring limit the maximum achievable frequency.

In order to enhance the achievable bandwidth of the amplifier in spite of these capacitances, an optimally valued inductor can be inserted to provide a resonant circuit with this capacitance. This technique, known as inductor peaking, is a fundamental technique that is commonly adopted for high-speed amplifier design [4]. As shown in Fig. 2, a typical RO-VCO can be modified by inserting an additional inductor $L$ within 


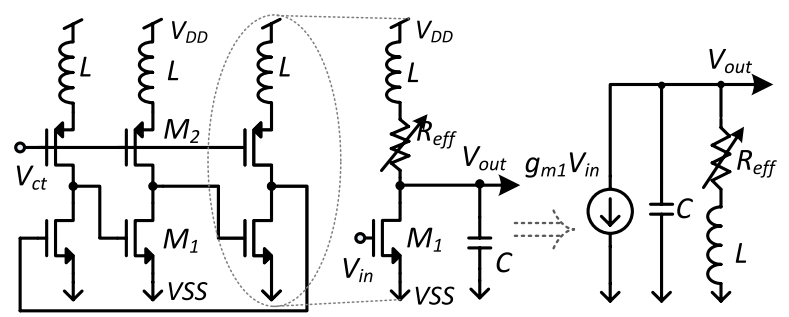

Fig. 2. Addition of peaking-inductor with RO-VCO.

TABLE I

PARAMETERs of Four Design Examples

\begin{tabular}{|c|c|c|c|c|}
\hline & \multicolumn{2}{|c|}{ IBM 130nm 8RF CMOS } & \multicolumn{2}{|c|}{ TSMC 65nm LP CMOS } \\
\hline & VCO-1 & VCO-2 & VCO-3 & VCO-4 \\
\hline $\mathrm{VDD}(\mathrm{V})$ & 1.5 & 1.5 & 1.2 & 1.2 \\
\hline $\mathrm{M} 1(\mathrm{~W} / \mathrm{L})(\mu \mathrm{m})$ & $22.5 / 0.12$ & $22.5 / 0.12$ & $20 / 0.06$ & $20 / 0.06$ \\
\hline $\mathrm{M} 2(\mathrm{~W} / \mathrm{L})(\mu \mathrm{m})$ & $72 / 0.12$ & $72 / 0.12$ & $64 / 0.06$ & $64 / 0.06$ \\
\hline Inductance $(\mathrm{pH})$ & $\approx 590$ & $\approx 420$ & $\approx 350$ & $\approx 350$ \\
\hline Peak Q@GHz & $18.3 @ 12$ & $7.2 @ 34$ & $21.5 @ 26$ & 12.5@33 \\
\hline
\end{tabular}

each delay cell, with the resulting transfer function given in (2):

$$
H(s)=\frac{V_{\text {out }}}{V_{\text {in }}}=\frac{-g_{m 1}\left(R_{\text {eff }}+s L\right)}{s^{2} C L+s C R_{\text {eff }}+1}
$$

Re-organizing (2) gives (3)

$$
\frac{V_{\text {out }}}{V_{\text {in }}}=-g_{m 1} R_{e f f} \frac{s+2 \zeta \omega_{n}}{s^{2}+2 \zeta \omega_{n} s+\omega_{n}^{2}} \cdot \frac{\omega_{n}}{2 \zeta}
$$

Where the nature frequency $\omega_{n}$ and damping factor $\zeta$ are given by (4)

$$
\omega_{n}=1 / \sqrt{L C} ; \zeta=\left(R_{e f f} / 2\right) \cdot \sqrt{C / L}
$$

It is interesting to note that (4) reveals two important facts that can guide the design of inductor-peaked RO-VCOs. First, for a fixed damping factor $\zeta$, the inductance $L$ is proportional to the capacitance $C$. Thus for an advanced CMOS process node which has smaller gate capacitance, the required peaking inductance could be reduced proportionally, which results in smaller silicon area with a more compact design. Secondly, for a given design, the smallest (worst) damping factor $\zeta$ occurs at the lowest value of $R_{e f f}$, which is also the point at which the VCOs operate at their highest frequency. Therefore, decreasing the frequency (frequency-tuning mechanism) has little negative effect on the loop stability. Furthermore, for practical implementation of on-chip monolithic inductors, there is usually a concern about the trade-off between the quality factor $(Q)$ of the inductor and the physical inductor size. We show later in this letter that the size of the inductor may influence the frequency range, whereas the $Q$ may dominate the phase noise performance of proposed RO-VCO.

\section{EXPERIMENTAL RESULTS}

To validate the proposed design approach, four design examples were fabricated at two different process nodes with the key parameters listed in Table I.

The key difference is the inductors realized in VCO-1/3 only use the top ultra-thick metal layer whereas the inductors realized on VCO-2/4 use stacked inductor structure on inner thinner layer, which results in a lower quality factor $(Q)$
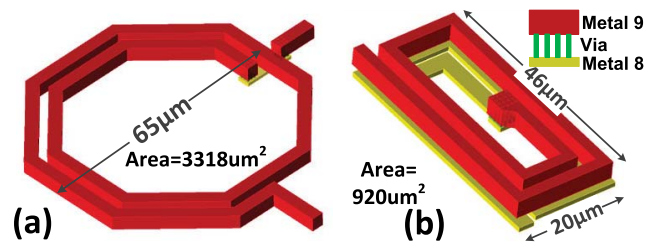

Fig. 3. Layout view of the inductors used with VCO-3(a) and VCO-4(b).

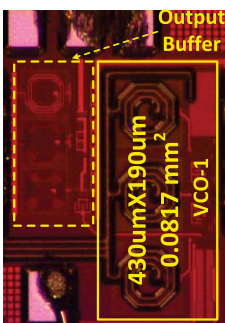

(a)

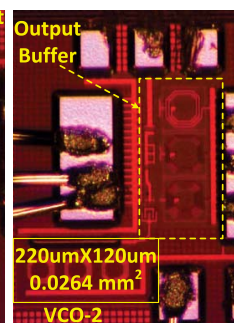

(b)

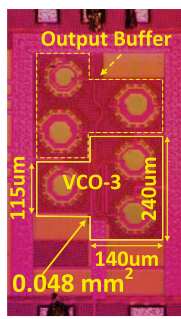

(c)

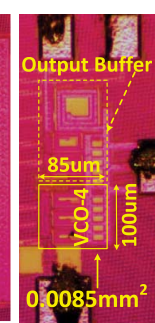

(d)
Fig. 4. Micoscope view of the proposed four RO-VCO designs.
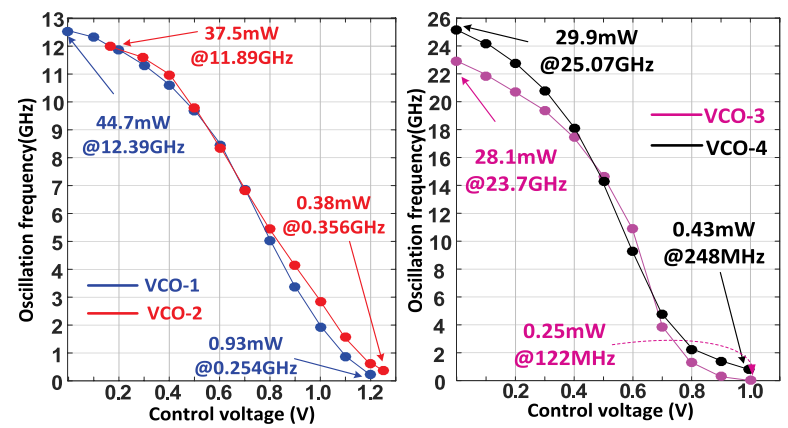

Fig. 5. Measured frequency tuning characteristics.

and a smaller silicon area. As illustrated in Fig. 3, the stacked inductor occupies less than one-third of silicon area compared to the inductor used with VCO-3, although the cost in performance is a relatively lower $Q$ factor. Micrographs of the four design examples are shown in Fig. 4. It can be seen that the VCOs realized in the $65 \mathrm{~nm}$ process node have much smaller silicon area than the corresponding designs realized in $130 \mathrm{~nm}$ process node. This is consistent with the theoretical prediction that the value of peaking inductor should be smaller in the advanced process node.

The output RF signal from each design example was fed into Keysight E4446A spectrum analyser. In summary, the measured voltage-frequency tuning characteristics of each design example are shown in Fig. 5, with the power consumption highlighted at the extremes of the tuning range. With the premise that identical transistor sizes were used in each pair of design examples, it is reasonable that they should have similar frequency-voltage tuning range characteristics. However, as highlighted in Fig. 6, there is a considerable difference when comparing the highest oscillation frequency of VCO-3 and VCO-4. The reason is that the large inductors used with VCO-3 indeed extend the signal wiring between each delay cell, which itself introduces a considerable additional parasitic capacitance, while the stacked-inductors used with VCO-4 eliminate this issue. This implies that using a stackedinductor not only reduces the overall silicon area, but can also 


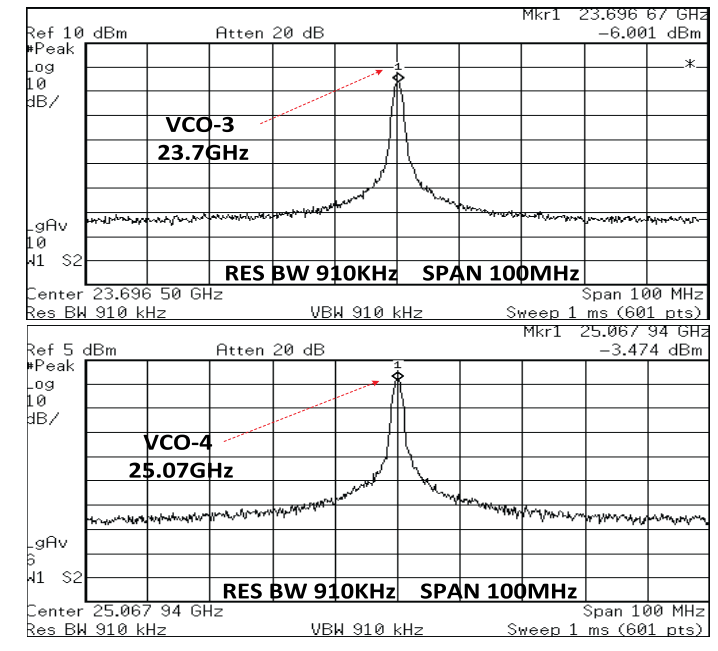

Fig. 6. The spectrum of highest oscillation frequency of VCO-3/4.

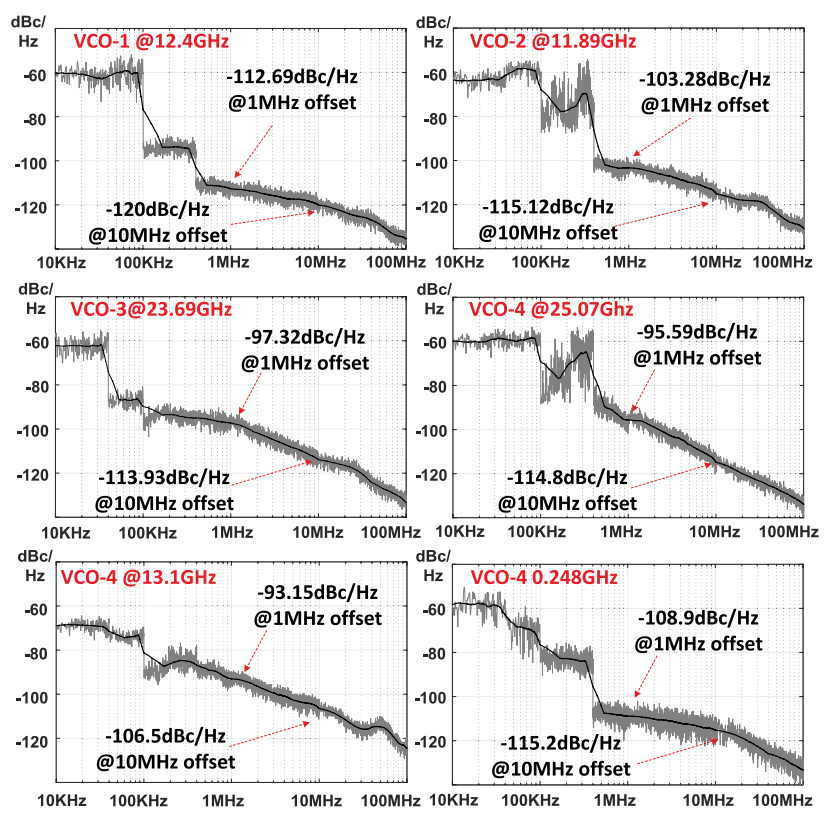

Fig. 7. Measured phase noise results of each design example.

benefit the tuning range of the proposed VCO by having an intrinsically lower parasitic capacitance.

As shown in Fig. 7, the measured phase noise results of each design example at its highest oscillation frequency are plotted. In additional to this, the phase noise performances of VCO-4 at its middle and lowest oscillations frequency are provided. Although a battery based power supply system is used to provide a clean power source, some environmental interference still can be observed at several hundred $\mathrm{KHz}$; but this does not prevent two conclusions can be obtained from these measurement figures. First, when tunning the oscillation frequency, phase noise performance does get slightly worse in the middle range, which was suppose due to the fact that inductor has smaller quality factor $(Q)$ at this lower frequency range. Secondly, it can be observed that both VCO-1/3 (big size) show better phase noise performance than their counterpart
TABLE II

PERFormanCE SUMMARY AND COMPARISON

\begin{tabular}{|c||c|c|c|c|c|}
\hline & {$[5]$} & {$[6]$} & {$[7]$} & VCO-2 & VCO-4 \\
\hline Process & $65 \mathrm{~nm}$ & $130 \mathrm{~nm}$ & $180 \mathrm{~nm}$ & $130 \mathrm{~nm}$ & $65 \mathrm{~nm}$ \\
\hline FTR range & $120 \%$ & $161 \%$ & $25.6 \%$ & $189 \%$ & $196 \%$ \\
$(\mathrm{~Hz})$ & $2 \mathrm{G}-8 \mathrm{G}$ & $1 \mathrm{G}-$ & $10 \mathrm{G}-$ & $0.36 \mathrm{G}-$ & $0.25 \mathrm{G}-$ \\
& $9.4 \mathrm{G}$ & $13.5 \mathrm{G}$ & $11.9 \mathrm{G}$ & $25 \mathrm{G}$ \\
\hline PDC(mW) & 6.4 & 3.7 & 2.4 & 37.5 & 29.9 \\
\hline PN & $-101 @$ & $-112 @$ & $-104.5 @$ & $-103.3 @$ & $-95.6 @$ \\
$\mathrm{dBc} / \mathrm{Hz} @$ & $1 \mathrm{M}$ & $10 \mathrm{M}$ & $1 \mathrm{M}$ & $1 \mathrm{M}$ & $1 \mathrm{M}$ \\
$\mathrm{f}_{\text {off }} / \mathrm{f}_{\text {osc }}(\mathrm{Hz})$ & $/ 4.2 \mathrm{G}$ & $/ 6 \mathrm{G}$ & $/ 12.7 \mathrm{G}$ & $/ 11.89 \mathrm{G}$ & $/ 25.07 \mathrm{G}$ \\
\hline FOM & 187 & 186 & 188.2 & $\mathbf{1 9 5 . 3}$ & $\mathbf{1 9 4 . 7}$ \\
\hline
\end{tabular}

VCO-2/4 (small size), which is supposed due to the fact that inductors used in VCO $1 / 3$ have higher quality factor than the inductors used in VCO-2/4. However, as illustrated in Table II, based on the normally used Figure of Merit (5) [5], these two small VCOs still demonstrate a considerable better performance when compared with the recent published results, provided that the smallest design only occupy $0.0085 \mathrm{~mm}^{2}$ silicon area

$$
\begin{aligned}
F O M= & -P N\left(f_{o f f}\right)+20 \log \frac{f_{\text {osc }}}{f_{\text {off }}}-10 \log \frac{P_{D C}}{1 m W} \\
& +20 \log \frac{F T R}{10}
\end{aligned}
$$

\section{CONCLUSION}

In this letter, we have demonstrated the feasibility of incorporating peaking inductors in Ring Oscillator VCOs. Experimental results show that the proposed circuit topology can take advantage of modern deep sub-micron processes, which may be used for clock generation and other similar applications in future advanced systems. The results indicate the best figure of merit $(\approx 195)$ in the field and the best combination of frequency and tuning range to the authors' knowledge. We would suggest that useful future work would be to use the symmetrical inductor with differential delay cells, and embed it in a PLL design.

\section{REFERENCES}

[1] S. Guo et al., "A low-voltage low-power $25 \mathrm{~Gb} / \mathrm{s}$ clock and data recovery with equalizer in 65 nm CMOS," in Proc. RFIC, May 2015, pp. 307-310.

[2] J. Jalil, M. B. I. Reaz, and M. A. M. Ali, "CMOS differential ring oscillators: Review of the performance of CMOS ROs in communication systems," IEEE Microw. Mag., vol. 14, no. 5, pp. 97-109, Aug. 2013.

[3] O. Momeni and E. Afshari, "High power terahertz and millimeter-wave oscillator design: A systematic approach," IEEE J. Solid-State Circuits, vol. 46, no. 3, pp. 583-597, Mar. 2011.

[4] B. Razavi, Design of Integrated Circuit for Optical Communications, 2nd ed. New York, NY, USA: Wiley, 2012.

[5] S. Yoo, J. J. Kim, and J. Choi, "A 2-8 GHz wideband dually frequencytuned ring-VCO with a scalable $\mathrm{K}_{\mathrm{VCO}}$," IEEE Microw. Wireless Compon. Lett., vol. 23, no. 11, pp. 602-604, Nov. 2013.

[6] C. Li et al., "A 1-9 GHz linear-wide-tuning-range quadrature ring oscillator in $130 \mathrm{~nm}$ CMOS for non-contact vital sign radar application," IEEE Microw. Wireless Compon. Lett, vol. 20, no. 1, pp. 34-36, Jan. 2010.

[7] T. P. Wang and Y.-M. Yan, "A low-voltage low-power wide-tuningrangehybrid class-AB/class-B VCO with robust start-up and highperformance $\mathrm{FOM}_{T}$," IEEE Trans. Microw. Theory Techn., vol. 62, no. 3, pp. 521-531, Mar. 2014. 